\title{
Single-centre retrospective review of risk factors for local tumour progression and complications in radiofrequency ablation of 555 hepatic lesions
}

\author{
Jasmine Ming Er $\underline{\text { Chua }}^{1}$, MBBS, frCR, Yu Ming Paul $\underline{\mathrm{Lam}}^{2}$, MBBS, Bien Soo $\underline{\operatorname{Tan}^{1}}$, MBBS, frCr, Kiang Hiong $\underline{\text { Tay }}{ }^{1}$, MBBS, frCR, \\ Apoorva Gogna ${ }^{1}$, MBBS, FRCR, Farah Gillan Irani ${ }^{1}$, MBBS, FRCR, Hoau Gong Richard Lo ${ }^{1}$, MBBS, FRCR, \\ Chow Wei $\underline{\text { TOO }}^{1}$, MBBS, FRCR
}

INTRODUCTION This study aimed to assess safety, local tumour progression (LTP) and risk factors for LTP after radiofrequency ablation (RFA) of liver tumours in a single centre.

METHODS All consecutive patients treated with RFA for liver tumours between January 2009 and October 2012 were included. Previously treated lesions that progressed were excluded. Using electronic medical records, the following data was captured: patient demographics, pre-procedural laboratory results, Child-Pugh status, tumour characteristics, development of tumoral seeding, RFA complications and LTP. Possible risk factors for LTP were identified using Cox regression.

RESULTS In total, 555 liver tumours were treated in 337 patients. 483 (87.0\%) hepatocellular carcinomas, 52 (9.4\%) colorectal metastases and 20 (3.6\%) other tumour types were treated. Mean tumour size was $2.1 \pm 1.1$ (range $0.4-6.8) \mathrm{cm}$. Mean follow-up duration was 387 days. 416 (75.0\%) lesions had no LTP at the last imaging. 70 (12.6\%) patients had minor complications requiring observation, while $7(1.3 \%)$ patients had significant complications requiring prolonged hospitalisation or further interventions. Only one case of tumour seeding was detected. Using multivariate Cox regression, the following factors were statistically significant in predicting LTP: hilar location (relative ratio [RR] 3.988), colorectal metastases (RR 2.075), size (RR 1.290) and younger age (RR 0.982).

CONCLUSION RFA of liver tumours is safe and effective, with a low significant complication rate of $1.3 \%$. Hilar lesions are most prone to LTP, followed by lesions that were larger in size and colorectal metastases. $75.0 \%$ of patients showed no LTP at the last follow-up.

Keywords: complication, liver tumours, local tumour progression, radiofrequency ablation

\section{INTRODUCTION}

Radiofrequency ablation (RFA) has gained wide acceptance as an effective treatment option for patients with unresectable primary and secondary liver malignancies. RFA involves a high-frequency alternating current that induces ionic agitation, resulting in frictional heating and destruction of the tissue surrounding the electrode. ${ }^{(1)}$ Although RFA is generally considered to be minimally invasive and safe, it is associated with several complications, including haemorrhage, liver abscess, hepatic infarction, liver failure, haemothorax, pleural effusion and tumour seeding. ${ }^{(2-4)}$ The rates of these potential complications are relatively low, especially for major complications that may require prolonged hospitalisation or further intervention. ${ }^{(5-7)}$

However, local tumour progression (LTP) remains a serious issue after ablation therapies. LTP is defined by the appearance of tumour foci at the edge of the ablation zone after at least one contrast-enhanced follow-up study has documented adequate ablation and an absence of viable tissue in the target tumour and surrounding ablation margin using imaging criteria. ${ }^{(8)}$ In this study, we intended to (a) determine possible risk factors for LTP to evaluate the safety and efficacy of RFA in controlling hepatic malignancy in our centre; and (b) review RFA-related complications and LTP.

\section{METHODS}

Informed consent was waived by the SingHealth Centralised Institutional Review Board. We retrospectively reviewed all consecutive cases of hepatic tumour treated with RFA performed in our centre from January 2009 to October 2012. Cases in which RFA was performed for LTP after prior ablation or in combination with transarterial chemoembolisation were excluded. The search yielded 555 hepatic tumours in 338 patients. Using the hospital's computerised records as well as cross-sectional imaging on the Picture Archiving and Communication System, the following data was captured: patient demographics; tumour type; length of follow-up; location and size of tumour; route of needle insertion; and assisted techniques used, if indicated (e.g. induced artificial ascites, catheterisation of vessels with balloon inflation to prevent heat sink, lipiodol localisation and intraprocedural contrast-enhanced ultrasonography for localisation). We also recorded patients' international normalised ratio, partial thromboplastin time (PTT), platelet count and Child-Pugh score. If the tumour occupied more than one segment of the liver, the

${ }^{1}$ Vascular and Interventional Radiology, Singapore General Hospital, Singapore, ${ }^{2}$ Faculty of Medicine, Baringa Private Hospital, Coffs Harbour, Australia Correspondence: Dr Ming Er Jasmine Chua, Associate Consultant, Vascular and Interventional Radiology, Singapore General Hospital, Outram Road, Singapore 169608. jasmine.chua.m.e@singhealth.com.sg 


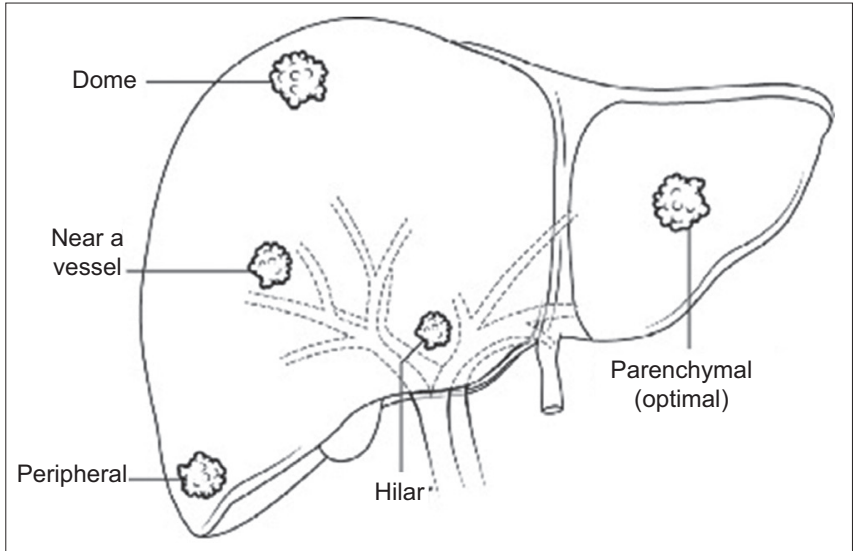

Fig. 1 Diagram shows tumour locations.

segment that contained the bulk of the tumour was selected. We used the largest diameter to denote tumour size, on either the axial or coronal planes. The route of needle insertion was determined using computed tomography $(\mathrm{CT})$ performed during the procedure.

We classified tumour location as (a) optimal location: surrounded by liver parenchyma and $>1 \mathrm{~cm}$ away from nearby structures; (b) dome lesion: $\leq 1 \mathrm{~cm}$ from the hepatic dome; (c) peripheral lesion: $\leq 1 \mathrm{~cm}$ from the hepatic capsule in a location other than the hepatic dome; (d) hilar lesion: $\leq 1 \mathrm{~cm}$ from the contents of the porta hepatis; and (e) lesion near a vessel: $\leq 1 \mathrm{~cm}$ from a vessel of diameter $>3 \mathrm{~mm}$ (Fig. 1). Tumour location was determined by a single reader. All peripheral lesions at the dome were classified as dome lesions. Lesions at the hilum or dome, which were near significant vessels, were classified as hilar or dome lesions.

The procedures were performed under ultrasonography or CT guidance, or a combination of both. Following department protocol, patients with platelet levels $<100,000 / \mu \mathrm{L}$ were transfused 4 units of random donor platelets or 1 unit of cryosupernatant. Likewise, patients with abnormal coagulation profiles (prothrombin time $[\mathrm{PT}]>13.2$ seconds, $\mathrm{PTT}>40.1$ seconds) were given 1 unit of fresh frozen plasma before the procedure. All RFAs were performed using a 17-gauge cooled-tip electrode (Cool-Tip ${ }^{\text {TM; }}$ RF Ablation System, Covidien, Minneapolis, MN, USA). RFA was performed as per standardised ablation protocol, with operators placing one or more needles simultaneously per ablation depending on tumour size. In larger lesions (> $2.5 \mathrm{~cm}$ ), we performed overlapping ablations with multiple switching electrodes (Cool-Tip; RF Ablation Switching Controller, Covidien, Minneapolis, MN, USA). In general, one electrode was used for lesions measuring 1-2 cm, two electrodes for lesions measuring 2-3 cm, and three or more electrodes for lesions larger than $3 \mathrm{~cm}$. For lesions near large vessels, notably the hilar lesions, caution was exercised to avoid vessel penetration by the radiofrequency (RF) electrode. We accessed dome lesions either via a subcostal, transhepatic, steep cranial angulated approach or directly, using an intercostal approach without traversing the lung or pleura. Multiphasic, contrast-enhanced CT was performed within 48 hours in most cases to assess for adequacy of ablation.
Complications were recorded and classified with Society of Interventional Radiology Standards of Practice Committee guidelines. ${ }^{(9)}$ Minor complications were classified as A (no therapy, no consequence) or B (nominal therapy, no consequence, including overnight admission for observation only). Major complications were classified as follows: C (required therapy, minor hospitalisation of $<48$ hours); D (required major therapy, unplanned increase in level of care, with prolonged hospitalisation of $>48$ hours); E (permanent adverse sequelae); or $\mathrm{F}$ (resulted in death).

Follow-up was considered finalised at death or the latest CT or magnetic resonance imaging documented before 28 September 2012. Transplant patients were censored from this study at the date of transplantation. Statistical analysis was performed using IBM SPSS Statistics for Windows, version 21.0 (IBM Corp, Armonk, New York, USA).

\section{RESULTS}

Patient and tumour characteristics are outlined in Table I. Out of 555 hepatic tumours that were ablated, 483 (87.0\%) were hepatocellular carcinomas (HCCs), 52 (9.4\%) were colorectal metastases and 20 (3.6\%) were other tumours. Mean tumour size was $2.1 \pm 1.1$ (range $0.4-6.8$ ) cm, and mean follow-up duration was 387 days. A total of $416(75.0 \%)$ lesions demonstrated no evidence of local tumour progression at the last imaging. Among the other 139 (25.0\%) lesions showing LTP, 54 (9.7\%) were detected within 100 days.

Table Il summarises the post-procedure complications that we encountered. There were 70 (12.6\%) minor complications and 7 (1.3\%) major complications. Major complications comprised three cases of haemoperitoneum, two hepatic abscesses, one pleural effusion and one death (shown in post-mortem to be due to an aortic dissection that was likely unrelated to the procedure). Minor complications comprised hydropneumothorax, ascites, bile duct injury, hepatic vessel injury, gastrointestinal injury and haematoma. Only one case of tumoral seeding was detected.

The results of multivariate Cox regression are shown in Table III. The following factors were identified to be significant in predicting LTP: hilar location (relative risk [RR] 3.988), colorectal metastases (RR 2.075), tumour size (RR 1.290) and the patient's age (RR 0.982). The lesions near the dome of the liver demonstrated a trend for higher LTP compared to the other locations (RR 1.796; $\mathrm{p}=0.053)$.

\section{DISCUSSION}

Reported LTP rates after RF ablation of hepatic tumour vary widely from $3 \%$ to $47 \%$ in the literature. ${ }^{(10-15)}$ Our rate of $25.0 \%$ certainly falls within the range of reported LTP and supports the use of RFA as an effective treatment for liver tumours. Previous studies have consistently demonstrated that tumour size is a significant factor in predicting LTP after RFA. ${ }^{(10,11,15-19)}$ Tumour size played a significant role in contributing to local progression in our study as well (RR 1.290; $p=0.017$ ), highlighting the importance of using size as a criterion when evaluating a patient's suitability to undergo RFA for hepatic malignancy. 
Table I. Baseline characteristics of the $\mathbf{3 3 8}$ patients who had lesions $(n=555)$.

\begin{tabular}{|c|c|}
\hline Characteristic & No. (\%) \\
\hline Male & $416(75.0)$ \\
\hline \multicolumn{2}{|l|}{ Type of tumour } \\
\hline $\mathrm{HCC}$ & $483(87.0)$ \\
\hline $\mathrm{mCRC}$ & $52(9.4)$ \\
\hline Others & $20(3.6)$ \\
\hline Follow-up duration* (day) & $387.1 \pm 331.4(0-1368)$ \\
\hline Size* $\left.^{*} \mathrm{~cm}\right)$ & $2.1 \pm 1.1(0.4-6.8)$ \\
\hline$<2$ & $315(56.8)$ \\
\hline $2-2.9$ & $142(25.6)$ \\
\hline $3-4.9$ & $89(16.0)$ \\
\hline$\geq 5$ & $9(1.6)$ \\
\hline \multicolumn{2}{|l|}{ Child-Pugh score } \\
\hline A & $419(75.5)$ \\
\hline B & $123(22.2)$ \\
\hline C & $13(2.3)$ \\
\hline INR* & $1.09 \pm 0.14(0.89-2.87)$ \\
\hline PTT* & $28.6 \pm 3.2(13.4-46.2)$ \\
\hline \multicolumn{2}{|l|}{ Segment } \\
\hline 1 & $7(1.3)$ \\
\hline 2 & $61(11.0)$ \\
\hline 3 & $47(8.5)$ \\
\hline $4 \mathrm{~A}$ & $60(10.8)$ \\
\hline $4 B$ & $28(5.0)$ \\
\hline 5 & $72(13.0)$ \\
\hline 6 & $86(15.5)$ \\
\hline 7 & $72(13.0)$ \\
\hline 8 & $122(22.0)$ \\
\hline Ascites present & $67(12.1)$ \\
\hline \multicolumn{2}{|l|}{ Assisted techniques } \\
\hline None & $510(91.9)$ \\
\hline Artificial ascites & $29(5.2)$ \\
\hline Balloon occlusion & $11(2.0)$ \\
\hline Lipoidal localisation & $2(0.4)$ \\
\hline Others & $3(0.5)$ \\
\hline \multicolumn{2}{|l|}{ Location } \\
\hline Optimal location & $114(20.5)$ \\
\hline Peripheral lesion & $214(38.6)$ \\
\hline Dome lesion & $104(18.7)$ \\
\hline Near hilar lesion & $23(4.1)$ \\
\hline Near vessel & $100(18.0)$ \\
\hline No local tumour progression & $416(75.0)$ \\
\hline
\end{tabular}

*Data presented as mean \pm standard deviation (range). HCC: hepatocellular carcinoma; INR: international normalised ratio; mCRC: metastatic colorecta cancer; PTT: partial thromboplastin time

Few papers in the published literature have evaluated the importance of tumour location in LTP, and there is no standardised method of classifying tumour location. ${ }^{(19-21)}$ Our study revealed that a hilar location (RR 3.988; $p=0.001)$ is more important than tumour size in contributing to LTP. This finding is in agreement with that of a recent paper by Toshimori et al, ${ }^{(20)}$
Table II. Classification of complications by grade.*

\begin{tabular}{|llllllll|}
\hline Complication & A & B & C & D & E & F & Total \\
\hline Abscess & 0 & 0 & 2 & 0 & 0 & 0 & 2 \\
\hline Haemoperitoneum & 17 & 0 & 1 & 2 & 0 & 0 & 20 \\
\hline Pleural effusion & 20 & 0 & 1 & 0 & 0 & 0 & 21 \\
\hline Hydropneumothorax & 2 & 0 & 0 & 0 & 0 & 0 & 2 \\
\hline Ascites & 17 & 0 & 0 & 0 & 0 & 0 & 17 \\
\hline Haemothorax & 2 & 0 & 0 & 0 & 0 & 0 & 2 \\
\hline Bile duct injury & 3 & 0 & 0 & 0 & 0 & 0 & 3 \\
\hline Hepatic vessel injury & 3 & 0 & 0 & 0 & 0 & 0 & 3 \\
\hline Colonic injury & 3 & 0 & 0 & 0 & 0 & 0 & 3 \\
\hline Gastric injury & 1 & 0 & 0 & 0 & 0 & 0 & 1 \\
\hline Skin haematoma & 2 & 0 & 0 & 0 & 0 & 0 & 2 \\
\hline Aortic injury & 0 & 0 & 0 & 0 & 0 & 1 & 1 \\
\hline Total & 70 & 0 & 4 & 2 & 0 & 1 & \\
\hline
\end{tabular}

*According to Society of Interventional Radiology Standards of Practice Committee classification.

whose classification of tumour location is the most detailed and similar to ours, compared to other published literature. ${ }^{(19,21)}$ We feel that a more detailed classification of tumour location may be the key to correct assessment of RFA efficacy within different regions of the liver.

The reason for the higher rate of LTP at the hilar region may be explained by the fact that needle placement in this area is more difficult for fear of injuring hilar structures, as well as the presence of large vessels that may lead to a heat-sink effect, resulting in less satisfactory ablations. The same reasons likely explain why dome lesions trended towards higher LTP. Our findings also suggest that with maturation of RFA technology, adequate ablations of larger lesions (within limits) may have already been achieved by the currently accepted method of multiple needle ablations. Perhaps lesions near the hilum may be better treated by other ablative methods such as irreversible electroporation ${ }^{(22,23)}$ or cryoablation. ${ }^{(24)}$

It was previously reported that tumours in certain locations may not be suitable for RFA, such as those adjacent to large vessels, due to a heat-sink effect resulting in incomplete ablation, or a nodule near the hilum or extrahepatic organs, due to the fear of injuring adjacent structures. ${ }^{(7,16,19)}$ Huang et al found that LTP was more frequently observed in 'dangerous locations', defined by locations that are less than $5 \mathrm{~mm}$ away from large blood vessels or extrahepatic organs $(46.3 \%$ vs. $22.2 \%$ for nodules in general locations). ${ }^{(25)}$ Contrary to other studies, we did not demonstrate significantly higher LTP for lesions that were in close proximity to major blood vessels but outside the hilar or dome regions. Electrode trajectory relative to the axis of the vessel may be a factor worth exploring. Interestingly, results from a study by Huang ${ }^{(26)}$ showed that the risk of incomplete ablation and vessel damage can be reduced by inserting the electrode orthogonal to, rather than parallel to, the vessel.

We found that colorectal metastases had a higher risk for LTP following RFA compared to other tumour types (RR 2.075; $p=0.0006$ ). Current literature on the impact of tumour 
Table III. Cox regression for local tumour progression.

\begin{tabular}{|lll|}
\hline Parameter & Hazard ratio & p-value \\
\hline Platelet & 0.998 & 0.163 \\
\hline Child-Pugh score & & 0.451 \\
\hline A & reference & \\
\hline B & 1.311 & 0.292 \\
\hline C & 0.665 & 0.584 \\
\hline INR & 0.985 & 0.982 \\
\hline Tumour segment & & 0.546 \\
\hline 1 & reference & \\
\hline 2 & 0.522 & 0.285 \\
\hline 3 & 0.388 & 0.115 \\
\hline $4 a$ & 0.668 & 0.475 \\
\hline $4 b$ & 0.339 & 0.142 \\
\hline 5 & 0.450 & 0.182 \\
\hline 6 & 0.372 & 0.095 \\
\hline 7 & 0.654 & 0.467 \\
\hline 8 & 0.607 & 0.359 \\
\hline Size (largest diameter) & 1.290 & 0.017 \\
\hline Location & & 0.004 \\
\hline Optimal location & reference & \\
\hline Peripheral & 1.114 & 0.692 \\
\hline Dome & 1.796 & 0.053 \\
\hline Hilar & 3.988 & 0.001 \\
\hline Vessel & 1.170 & 0.591 \\
\hline No. of burns per lesion & 1.251 & 0.026 \\
\hline Male gender & 1.339 \\
\hline Age & 0.982 & 0.021 \\
\hline Histological type & & \\
\hline HCC & reference & \\
\hline mCRC & 2.075 & \\
\hline Others & & \\
\hline & & \\
\hline
\end{tabular}

HCC: hepatocellular carcinoma; INR: international normalised ratio mCRC: metastatic colorectal cancer

histology on LTP is controversial. In a large study of 1,032 lesions, Berber and Siperstein ${ }^{(10)}$ reported that LTP was the highest for colorectal metastasis (34\%), followed by non-colorectal, non-neuroendocrine metastasis (22\%); HCC (18\%); and neuroendocrine metastasis (6\%). Previous studies have attributed the improved heating efficacy in RFA of HCC ('oven effect') to the lower thermal conductivity of the surrounding cirrhotic liver parenchyma. ${ }^{(27,28)}$ Bleicher et al, however, identified a higher local recurrence rate for HCCs compared to metastasis from colorectal malignancy. ${ }^{(29)}$ In another study, Chow et al concluded that tumour type (HCC vs. liver metastasis) confers no significant impact on local recurrence. ${ }^{(13)}$ Hence, the significance of tumour type for LTP is yet uncertain. Future investigations can help to elucidate whether a particular type of tumour is more resistant to RFA.

An interesting result from our study was the slightly lower risk of LTP conferred by older age (RR 0.982; $p=0.026)$. Yu et al previously reported that patients with early tumour recurrence after RFA were younger than the patients without recurrence. ${ }^{(12)}$
To the best of our knowledge, no study has yet investigated the inverse relationship between age and local tumour recurrence following ablation. Compared to older patients, younger patients may have superior cellular mechanisms with a greater ability to reproduce and proliferate, which could contribute to the aggressiveness of a tumour. The mechanism that results in this small but potentially important finding may warrant evaluation in future molecular studies, particularly in the era of individualised oncologic therapy for HCCs. Nevertheless, the RR for age is still close to 1 and may not carry significant clinical impact.

In our study, there were 70 (12.6\%) minor complications and $7(1.3 \%)$ major complications, one of which $(0.2 \%)$ resulted in death. Our result is comparable to reports from other centres. ${ }^{(5-7,30)}$ An Italian multicentre study of 3,500 ablated lesions quoted rates of major complications and deaths of $2.2 \%$ and $0.3 \%$ respectively. ${ }^{(7)}$ Chen et al reported a major complication rate of $5.2 \%$ based on 183 tumours ablated. ${ }^{(5)}$ De Baère et al also reported a similar figure, with major complications occurring in $5.7 \%$ of the total lesions treated; ${ }^{\left({ }^{(6)}\right.}$ procedure-related death occurred in $1.4 \%$ of the patients in the same study. A large Korean study on 3,000 lesions reported a procedure-related mortality rate of $0.15 \%$. ${ }^{(30)}$ Thus, our results based on 555 lesions over a threeyear period show a similar safety profile to the published reports, further confirming the role of RFA as a safe alternative treatment option for patients with hepatic tumours unsuitable for surgical resection or who declined surgery. Using Cox regression, we did not find any risk factor that predicted major complications, although this may have been confounded by the small number of major complications encountered.

Our study had several limitations. First, it is a retrospective study with no standardised follow-up. Second, interventional radiologists with varying experience levels performed ablation procedures, forming a possible source of bias. As the focus of this study was LTP, we did not look at distal recurrence or overall survival, which are important clinical measures. However, one can argue that distant recurrence may be due to a de novo HCC rather than an intrahepatic metastasis. The tumour locations of lesions adjacent to vessels at the dome and the hilar areas were preferentially classified as dome or hilar rather than vessel. Although this could have confounded our results, the intent of this classification was to reflect the overall real world difficulty in ablating at these locations. Also, there is no standardised way of classifying lesions based on location; as such, our results are not easily comparable with other similar studies. We also recognise that the number of patients with hilar tumours is small, which may lead to bias, but this factor, as well as interaction between the different parameters, should have been taken into account by the regression analysis.

In conclusion, RFA of liver tumours is safe and effective with a low major complication rate. We found that tumour location at the hilum was the strongest risk factor for LTP, followed by metastatic colorectal tumour type and larger lesion size. Future studies that use a standardised format for reporting tumour location may help to validate our findings. 


\section{ACKNOWLEDGEMENT}

We would like to acknowledge Miss Wei Yuan, Singapore Clinical Research Institute, Singapore, for her help in statistical analysis.

\section{REFERENCES}

1. Gazelle GS, Goldberg SN, Solbiati L, Livraghi T. Tumor ablation with radiofrequency energy. Radiology 2000; 217:633-46.

2. Koda M, Murawaki Y, Hirooka Y, et al. Complications of radiofrequency ablation for hepatocellular carcinoma in a multicenter study: an analysis of 16,346 treated nodules in 13,283 patients. Hepatol Res 2012; 42:1058-64.

3. Bertot LC, Sato M, Tateishi R, Yoshida H, Koike K. Mortality and complication rates of percutaneous ablative techniques for the treatment of liver tumors: a systematic review. Eur Radiol 2011; 21:2584-96.

4. Goto E, Tateishi R, Shiina S, et al. Hemorrhagic complications of percutaneous radiofrequency ablation for liver tumors. J Clin Gastroenterol 2010; 44:374-80.

5. Chen TM, Huang PT, Lin LF, Tung JN. Major complications of ultrasound-guided percutaneous radiofrequency ablations for liver malignancies: single cente experience. J Gastroenterol Hepatol 2008; 23(8 Pt 2):e445-50.

6. de Baère $T$, Risse $O$, Kuoch $V$, et al. Adverse events during radiofrequency treatment of 582 hepatic tumors. AJR Am J Roentgenol 2003; 181:695-700.

7. Livraghi T, Solbiati L, Meloni MF, et al. Treatment of focal liver tumors with percutaneous radio-frequency ablation: complications encountered in a multicenter study. Radiology 2003; 226:441-51.

8. Ahmed M, Solbiati L, Brace CL, et al; International Working Group on ImageGuided Tumor Ablation; Interventional Oncology Sans Frontières Expert Panel Technology Assessment Committee of the Society of Interventional Radiology; Standard of Practice Committee of the Cardiovascular and Interventional Radiological Society of Europe. Image-guided tumor ablation: standardization of terminology and reporting criteria--a 10-year update. J Vasc Interv Radio 2014; 25:1691-705.e4

9. Omary RA, Bettmann MA, Cardella JF, et al; Society of Interventional Radiology Standards of Practice Committee. Quality improvement guidelines for the reporting and archiving of interventional radiology procedures. J Vasc Interv Radiol 2003; 14(9 Pt 2):S293-5.

10. Berber E, Siperstein A. Local recurrence after laparoscopic radiofrequency ablation of liver tumors: an analysis of 1032 tumors. Ann Surg Oncol 2008, 15:2757-64

11. Lam VW, Ng KK, Chok KS, et al. Risk factors and prognostic factors of local recurrence after radiofrequency ablation of hepatocellular carcinoma. J Am Coll Surg 2008; 207:20-9.

12. Yu HC, Cheng JS, Lai KH, et al. Factors for early tumor recurrence of single small hepatocellular carcinoma after percutaneous radiofrequency ablation therapy. World J Gastroenterol 2005; 11:1439-44.

13. Chow DH, Sinn LH, Ng KK, et al. Radiofrequency ablation for hepatocellula carcinoma and metastatic liver tumors: a comparative study. J Surg Oncol 2006; 94:565-71.

14. Lee DH, Lee JM, Lee JY, et al. Radiofrequency ablation of hepatocellular carcinoma as first-line treatment: long-term results and prognostic factors in
162 patients with cirrhosis. Radiology 2014; 270:900-9.

15. Shiina S, Tateishi R, Arano T, et al. Radiofrequency ablation for hepatocellular carcinoma: 10-year outcome and prognostic factors. Am J Gastroenterol 2012; 107:569-77.

16. Nishikawa $\mathrm{H}$, Inuzuka $\mathrm{T}$, Takeda $\mathrm{H}$, et al. Percutaneous radiofrequency ablation therapy for hepatocellular carcinoma: a proposed new grading system for the ablative margin and prediction of local tumor progression and its validation. J Gastroenterol 2011; 46:1418-26.

17. N'Kontchou G, Mahamoudi A, Aout M, et al. Radiofrequency ablation of hepatocellular carcinoma: long-term results and prognostic factors in 235 Western patients with cirrhosis. Hepatology 2009; 50:1475-83.

18. Ayav A, Germain A, Marchal F, et al. Radiofrequency ablation of unresectable liver tumors: factors associated with incomplete ablation or local recurrence. Am J Surg 2010; 200:435-9.

19. Teratani T, Yoshida H, Shiina S, et al. Radiofrequency ablation for hepatocellular carcinoma in so-called high-risk locations. Hepatology 2006; 43:1101-8.

20. Toshimori J, Nouso K, Nakamura S, et al. Local recurrence and complications after percutaneous radiofrequency ablation of hepatocellular carcinoma: a retrospective cohort study focused on tumor location. Acta Med Okayama 2015; 69:219-26.

21. Murakami T, Ishimaru H, Sakamoto I, et al. Percutaneous radiofrequency ablation and transcatheter arterial chemoembolization for hypervascular hepatocellular carcinoma: rate and risk factors for local recurrence. Cardiovasc Intervent Radiol 2007; 30:696-704.

22. Chen X, Ren Z, Zhu T, et al. Electric ablation with irreversible electroporation (IRE) in vital hepatic structures and follow-up investigation. Sci Rep 2015; 5:16233.

23. Lencioni R, de Baere T, Martin RC, Nutting CW, Narayanan G. Image-guided ablation of malignant liver tumors: recommendations for clinical validation of novel thermal and non-thermal technologies: a Western perspective. Liver Cancer 2015; 4:208-14

24. Littrup PJ, Aoun HD, Adam B, et al. Percutaneous cryoablation of hepatic tumors: long-term experience of a large U.S. series. Abdom Radiol (NY) 2016; 41:767-80.

25. Huang JW, Hernandez-Alejandro R, Croome KP, et al. Surgical vs percutaneous radiofrequency ablation for hepatocellular carcinoma in dangerous locations. World J Gastroenterol 2011; 17:123-9.

26. Huang HW. Influence of blood vessel on the thermal lesion formation during radiofrequency ablation for liver tumors. Med Phys 2013; 40:073303.

27. Liu Z, Ahmed M, Weinstein Y, et al. Characterization of the RF ablationinduced 'oven effect': the importance of background tissue thermal conductivity on tissue heating. Int J Hyperthermia 2006; 22:327-42.

28. Livraghi T, Goldberg SN, Lazzaroni S, et al. Small hepatocellular carcinoma: treatment with radio-frequency ablation versus ethanol injection. Radiology 1999; 210:655-61.

29. Bleicher RJ, Allegra DP, Nora DT, et al. Radiofrequency ablation in 447 complex unresectable liver tumors: lessons learned. Ann Surg Oncol 2003; 10:52-8.

30. Rhim H, Lim HK, Kim YS, Choi D, Lee WJ. Radiofrequency ablation of hepatic tumors: lessons learned from 3000 procedures. J Gastroenterol Hepatol 2008; 23:1492-500. 